\title{
The challenge of managing a cetuximab rash
}

\author{
Samara E Pollock, BA, ${ }^{a}$ Lowell Shinn, MD, ${ }^{\mathrm{b}}$ Nancy Delman, RN,,${ }^{\mathrm{c}}$ Jeanne Fuscardo, RN, \\ and Jondavid Pollock, $\mathrm{MD}, \mathrm{PhD}^{\mathrm{c}}$
}

${ }^{a}$ Boston University School of Medicine, Boston, Massachusetts; and Divisions of ${ }^{\mathrm{b}}$ Medical Oncology and ${ }^{\mathrm{c}}$ Radiation Oncology, The Schiffler Cancer Center, Wheeling, West Virginia

$\mathrm{E}$ pidermal growth factor receptor antibodies (EGFR) such as cetuximab have been approved for use as first-line management as well as salvage therapy for head and neck and colorectal cancers. Among the most common expected toxicity is a cutaneous eruption described as acneiform. The presence of a rash has been postulated to predict a more favorable treatment outcome for cancers of the head and neck ${ }^{1}$ but not for colorectum. ${ }^{2}$ With more severe drug reactions, patients may require a treatment break, which has been shown to reduce locoregional control and survival, particularly in patients with head and neck cancer. ${ }^{3}$ This has prompted clinicians to affect rapid therapy to reverse the drug eruption. Given the controversy around rapid and effective reversal of this drug reaction, this report aims to address the current status of clinical management using an actual patient vignette.

\section{Case presentation and summary}

The patient was a 57-year-old white man who had been diagnosed with stage 4T4N0M1 grade 3 cutaneous squamous cell carcinoma (SCC) of the right postauricular soft tissues, with erosion into the right mastoid and biopsy-proven metastatic disease involving the contralateral left supraclavicular fossa and bilateral lungs. His disease became chemotherapy-refractory, and he was referred for palliative local therapy to the base of skull. Because of the size of the tumor $(4 \mathrm{~cm} \times 5 \mathrm{~cm})$, he was considered for sensitizing chemotherapy, but cisplatin was not appropriate because of chronic hearing loss. ${ }^{4}$ The patient was recommended sensitizing doses of cetuximab. This EGFR antibody has been shown to offer similar benefits to those seen with cisplatin in the definitive management of head and neck SCC. ${ }^{5}$
The standard loading dose of cetuximab was given at $400 \mathrm{mg} / \mathrm{m}^{2}$ intravenously (IV). The following week, the sensitizing dose of $250 \mathrm{mg} / \mathrm{m}^{2}$ IV was given along with daily radiotherapy to the target volumes. The weekly dose of cetuximab continued at $250 \mathrm{mg} / \mathrm{m}^{2}$. The radiotherapy prescription was for $6,000 \mathrm{cGy}$ in $200 \mathrm{cGy}$ daily fractions, encompassing the gross tumor volume as identified on a computed-tomographic scan with 3-mm cuts. We used a noncoplanar arc radiotherapy beam arrangement because it inherently spreads the dose over a larger volume of normal tissue while conformally delivering its largest dose to the gross tumor volume. As such, a volume of the patient's oropharynx and oral cavity was included within the radiotherapy dose penumbra. After receiving 3 weekly doses of cetuximab (1 loading dose and 2 weekly sensitizing doses) and 2,000 cGy of radiotherapy, the patient developed a robust grade 2 cutaneous eruption delimited to the face, with few scattered lesions on the upper anterior chest. He was seen in the medical oncology department and was prescribed doxycycline $100 \mathrm{mg}$ orally twice daily and topical clindamycin $2 \%$ ointment twice daily.

In the radiation oncology clinic, his drug therapy was manipulated. His cetuximab cutaneous reaction was a grade 2 , manifested by moderate erythema with nonconfluent moist desquamation. Because of concern that the patient would develop oral candida, which would further delay his therapy, the oral and topical antibiotics were discontinued, as was the oral prednisone. He was prescribed triamcinolone cream $0.1 \%$ to be applied to the facial and few chest wall areas twice daily and an oncology mouth rinse to address early nonconfluent mucositis. The accompanying images show the extent of the patient's cetuximab cutaneous reaction at baseline before

Accepted for publication November 27, 2018. Correspondence: Jondavid Pollock, MD, PhD; ipollock@wheelinghospital. org. The authors report no disclosures or conflicts of interest. JCSO 2018;16(6):e272-e276. doi: https://doi. org/10.12788/jcso.0440 

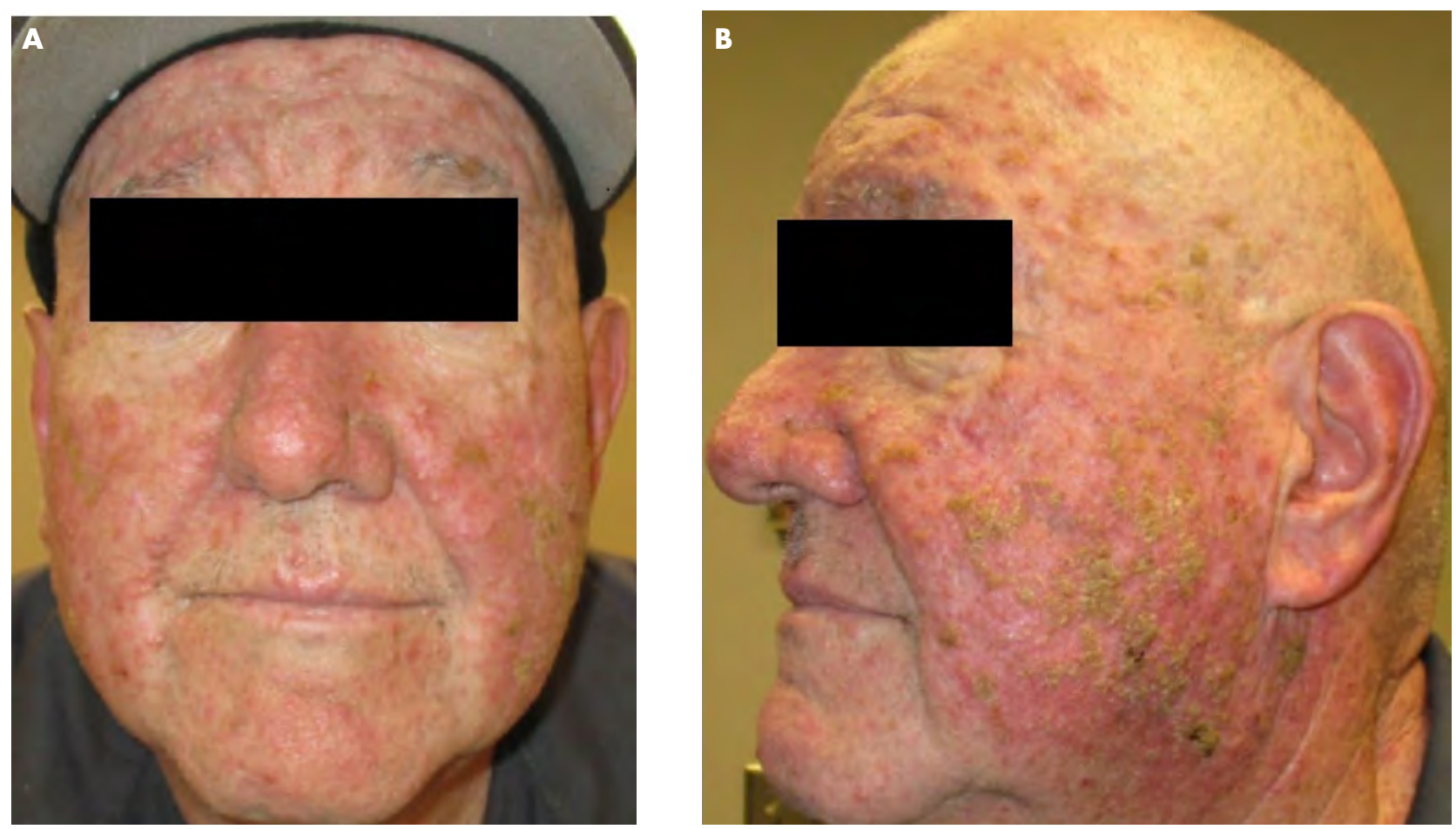

FIGURE 1 The patient at baseline, before treatment initiation for a robust grade 2 cetuximab cutaneous reaction manifested by moderate erythema with nonconfluent moist desquamation. A, front view, and B, side view.

treatment initiation (Figure 1), at 4 days after the intervention (Figure 2), and again at 6 days after the intervention (Figure 3). The patient consented to having his photographs taken and understood that they would be used for educational and research publication purposes.

As can be seen from the photographs, the patient's rash began to dry and peel by day 4 after the intervention, and there were no new eruptions. The pruritus that accompanied the rash had entirely resolved. By day 6 , the rash had completely subsided. Because of the response to the topical steroid, the patient continued cetuximab without a dose modification. He was recommended to continue with the triamcinolone cream until the chemoradiotherapy course concluded.

\section{Discussion}

A cetuximab-induced rash is common. In a 2011 metaanalysis quantifying grades 1 to 4 in severity, about $75 \%$ of patients treated with an EGFR inhibitor experienced a rash. Most of the rashes were lower than grade 3, and the drug was either dose-reduced or temporarily held, but it was not generally discontinued. ${ }^{6}$ Of note is that in a nonselected survey of medical oncologists who were prescribing cetuximab, $76 \%$ reported holding the drug owing to rash severity, $60 \%$ reported dose reductions for a drug rash, and $32 \%$ reported changing the drug because of rash severity. ${ }^{7}$

In the initial pharmaceutical registration trial, $76 \%$ to

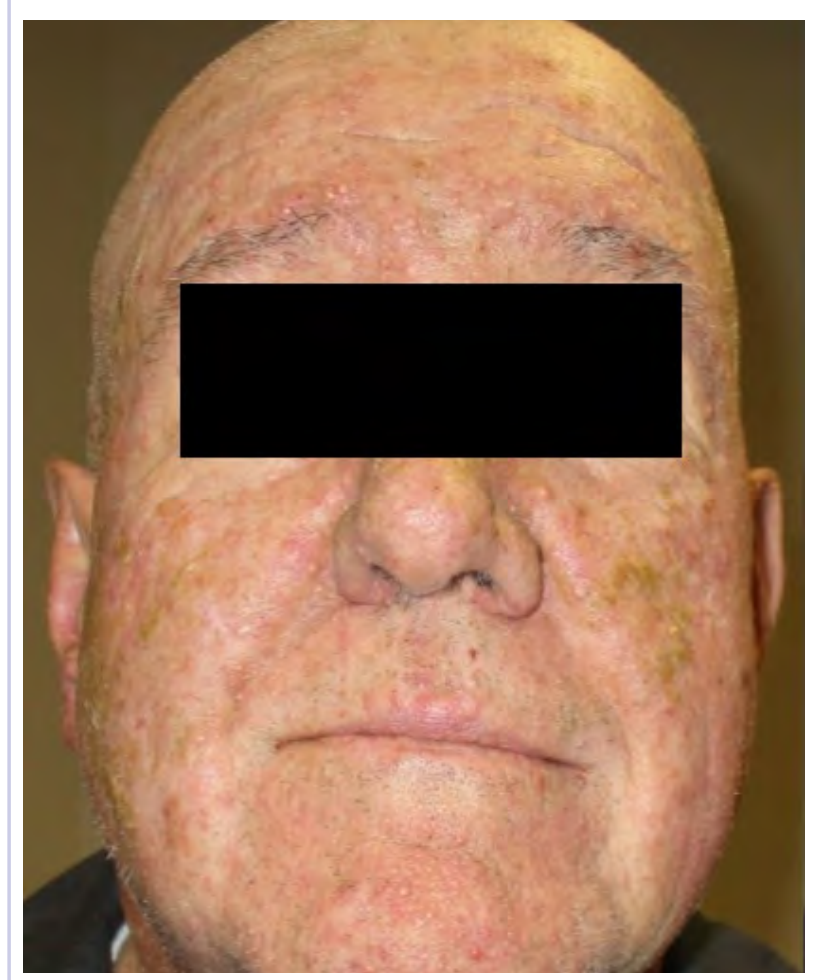

FIGURE 2 The patient 4 days after intervention with doxycycline $100 \mathrm{mg}$ orally twice daily and topical clindamycin $2 \%$ ointment twice daily for a grade 2 cetuximab cutaneous reaction. 

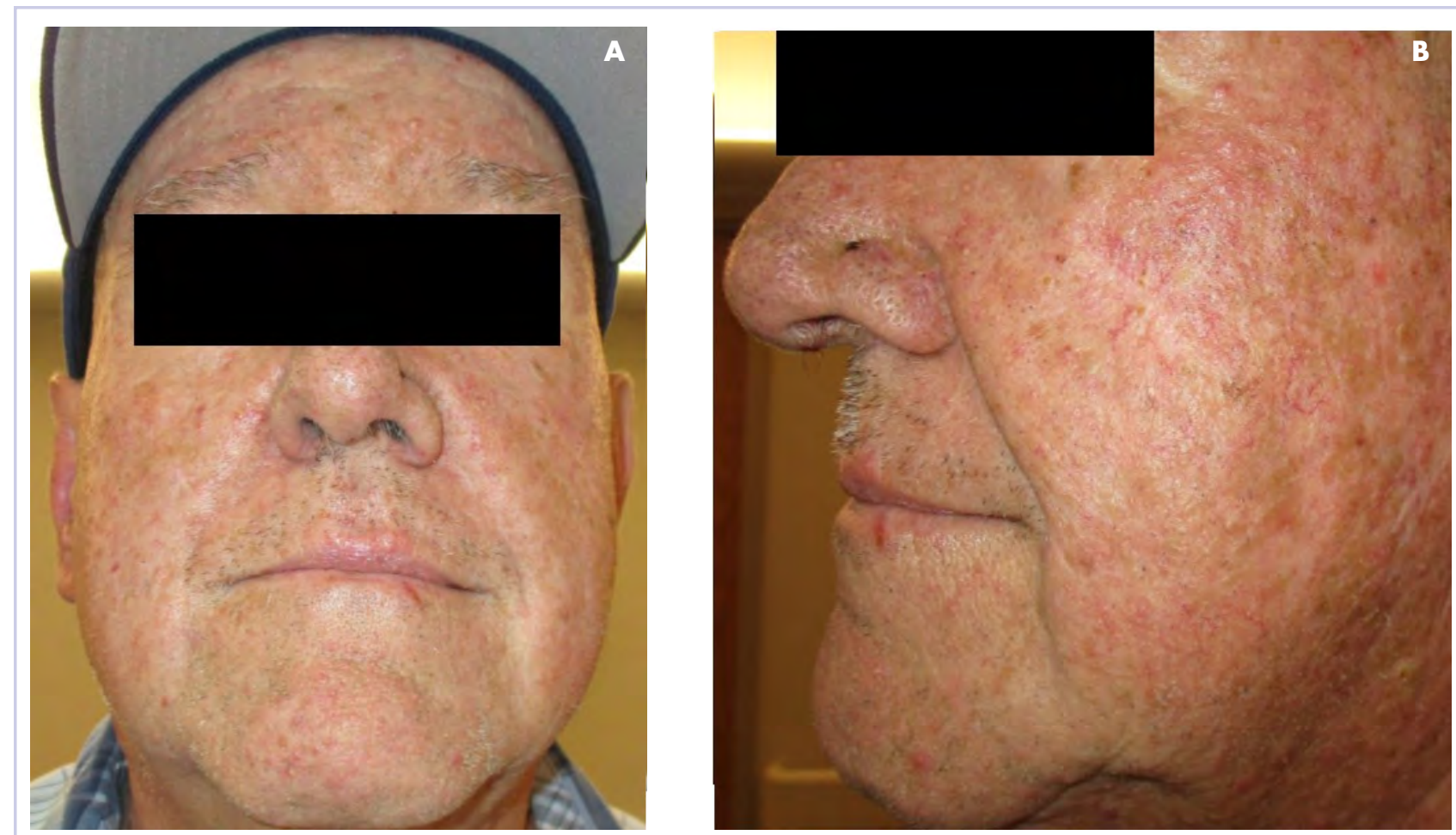

FIGURE 3 The patient 6 days after intervention with doxycycline $100 \mathrm{mg}$ orally twice daily and topical clindamycin $2 \%$ ointment twice daily for a grade 2 cetuximab cutaneous reaction. A, front view, and B, side view.

$88 \%$ of patients who received cetuximab developed a rash, $17 \%$ of which were at least grade 3 . The pharma recommendations for managing the drug rash include a drug delay for up to 2 weeks for a rash of grade 3 or less and to terminate use of the drug if there is no clinical improvement after 2 weeks. ${ }^{8}$ Biopsies of the rash confirm a suppurative inflammatory reaction separate from an infectious acne reaction, ${ }^{9}$ resulting in a recommendation to treat with topical steroid therapy. In some circumstances, the drug reaction can become infected or involve the paronychia, often related to Staphylococcus aureus. ${ }^{10}$ Despite what would otherwise be a problem addressed by anti-inflammatory medical therapy, the clinical appearance of the rash marked by pustules, coupled with the relative immunosuppressed state of a cancer patient, has prompted medical oncologists to prescribe antibiotic therapy.

To address the many single-institutional reports on management of the EGFR rash, several guidelines have been published. The earliest guideline - after a report that concurrent cetuximab and radiotherapy was superior to radiotherapy alone in locally advanced head and neck cancer, which documented a $23 \%$ incidence of at least grade 3 cutaneous toxicity in the cetuximab arm ${ }^{1}-$ attempted to score the severity of the rash according to the National Cancer Institute's (NCI) Common Terminology Criteria for Adverse Events (CTCAE). Under those criteria, the authors defined grade 2 toxicity as moderate to brisk ery- thema with patchy moist desquamation, mostly confined to skin folds and creases. Grade 3 toxicity was described as moist desquamation other than skin folds and creases with bleeding induced by minor trauma, and grade 4 skin toxicity was defined as skin necrosis or ulceration of full thickness dermis with spontaneous bleeding from the involved site. The authors went on to describe a grade-related treatment algorithm that included gently washing the skin, keeping it dry, and using topical anti-inflammatory agents, including steroids. Antibiotics should be used in the presence of a suspected infection after culturing the area, and grade 4 toxicity should be referred to a wound care center. ${ }^{11}$

In a consensus statement from the National Comprehensive Cancer Network, the authors noted that most management recommendations were anecdotal. They recommended against the use of astringents and other drying agents because they exacerbate pain. The ultimate choice of topical steroids or antibiotics was based entirely on subjective judgement given the absence of prospective data. ${ }^{12}$

A Spanish consensus conference report argued against any prophylaxis against a skin reaction, other than keeping the skin clean and dry. ${ }^{13}$ The authors of the report recommended against washing the affected skin more than twice a day to avoid excess drying, and they advocated for moisturizers and debridement of skin crusting with hydrogels to reduce superinfection and bleeding. ${ }^{13}$ The authors also noted that some guidelines have suggested that topi- 
cal steroids might exacerbate a skin rash, ${ }^{14}$ but they concluded that topical steroids are beneficial as long as they are used for less than 2 weeks. Any use of antibiotics should be based on clear evidence of an infection. ${ }^{13}$

In the first modification of the NCI's CTCAE rash grading scale, an international panel addressed the increasing number of reports in the literature suggesting that the previous toxicity scale was possibly inadequate in its recommendations for appropriate treatment. The initial scale had defined only the skin reaction and not what therapy should be administered; therefore, in the update, the descriptions for grades 1 and 2 toxicity remained unchanged, but oral antibiotics were recommended for grade 3 lesion, and parenteral antibiotics with skin grafting were required with grade 4 toxicity. ${ }^{15}$

An Asian expert panel suggested modifying the bioradiation dermatitis scale, defining a grade 3 dermatitis as $>50 \%$ moist desquamation of the involved field with formation of confluent lesions because of treatment. They recommended both topical and oral therapy, wound care, and possible hospitalization in severe cases. The panel suggested topical and systemic steroids and antibiotics. ${ }^{16}$

Finally, in an Italian consensus report, the members again modified the skin toxicity grading and were notably more aggressive in terms of their management recommendations. They defined grade 2 toxicity as pustules or papules covering $10 \%$ to $30 \%$ of the body surface area, with potential pruritus or tenderness. They also noted the psychosocial impact of skin toxicities on patients and the limits to their activities of daily living. They recommended vitamin K1 (menadione) cream, topical antibiotics, topical intermediate potency steroids, and oral antibiotic therapy for up to 4 weeks for grade 2 toxicity. Despite this aggressive treatment course, the authors admitted that the utility of topical steroids and antibiotics was unknown. They defined grade 3 toxicity as pustules or papules covering more than $30 \%$ of the body surface area, with signs of possible pruritus and tenderness. Activities of daily living and self-care were affected, and there was evidence of a superinfection. The panel suggested use of antibiotics pending culture results, oral prednisone, antihistamines, and oral analgesics. Topical therapy was not included. ${ }^{17}$ It is noteworthy that only the Italian panel recommended the use of vitamin K1 cream. In a prospective randomized, double-blinded, placebo-controlled phase 2 trial of 30 patients, menadione exhibited no clinical benefit in terms of reducing the severity of cetuximab skin lesions. ${ }^{18}$

Figure 4 illustrates our institutional approach to treating cetuximab rash based on a combination of the Spanish and NCI approaches.

The ultimate choice of therapy to manage a cetuximab

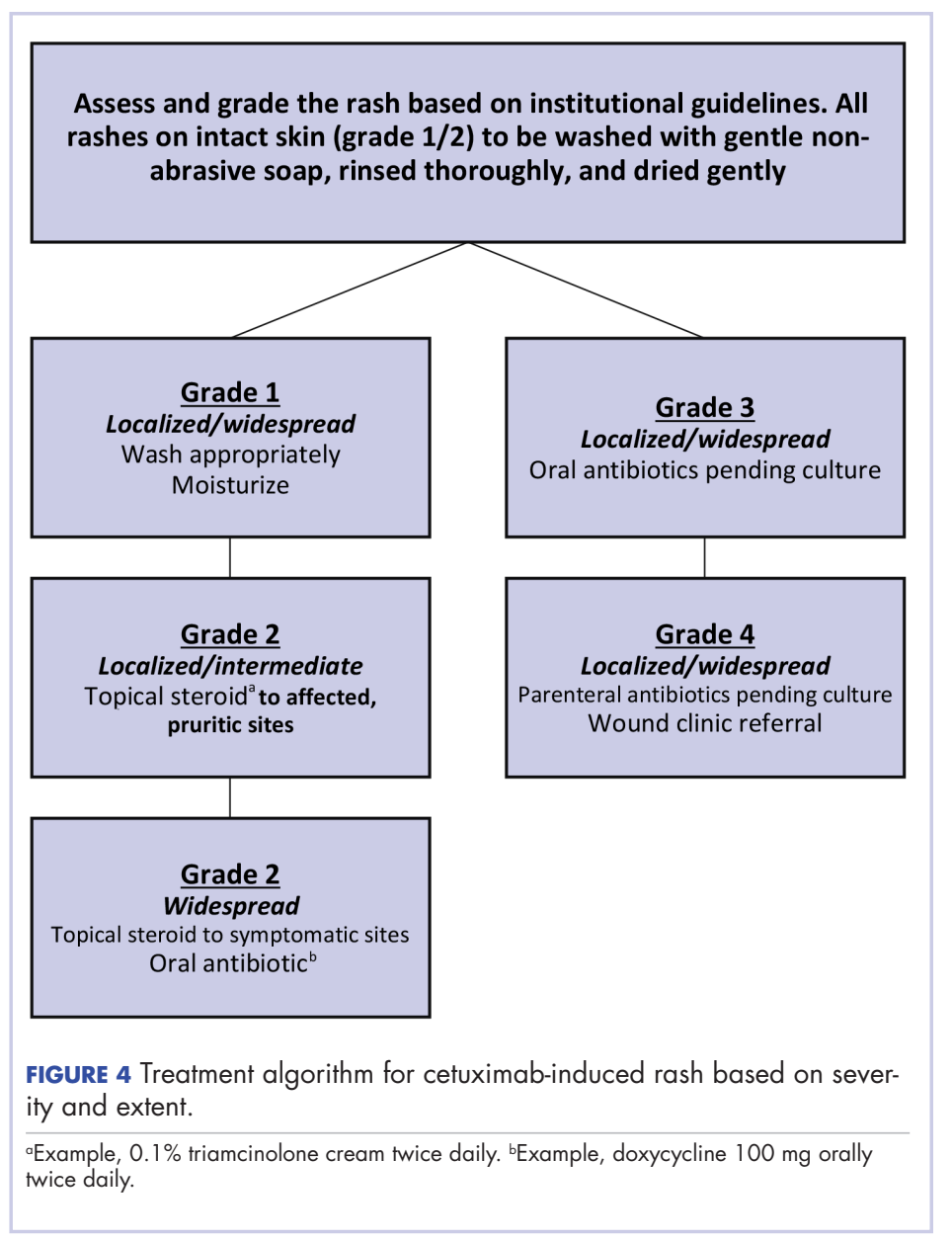

rash must be patient and treatment specific. Our institutional approach, like that of the Spanish series, ${ }^{13}$ is to avoid chemoprophylaxis against a rash; rather, we recommend daily washing of the skin with a gentle soap followed by thorough rinsing and adequate, nonaggressive drying. Moisturizing the intact skin has been shown to reduce exfoliation, and we have incorporated that approach into our regimen. ${ }^{19}$

In our patient, whose head and neck radiotherapy tumor volume included a portion of the oral cavity and oropharynx, systemic antibiotic and steroid therapy would likely lead to further complications with the development of oral candidiasis. Therefore, while the severity of the reaction remained a grade 2 , it seemed appropriate to treat with topical intermediate potency steroids and skin cleansing only. If the reaction had become more severe, then cultures would have been obtained to guide our decision on antibiotic therapy. Our patient's response to topical steroids was predictable and effective, and he was able to proceed with his course of cancer therapy. 


\section{References}

1. Bonner JA, Harari PM, Giralt J, et al. Radiotherapy plus cetuximab for locoregionally advanced head and neck cancer: 5-year survival data from a phase 3 randomised trial, and relation between cetuximabinduced rash and survival. Lancet Oncol. 2010;11(1):21-28.

2. Sommeijer DW, Karapetis CS, Zalcberg JR, et al. The relationship between rash, tumor mutation KRAS status and clinical and quality of life outcomes in patients with advanced colorectal cancer treated with cetuximab in the NCIC CTG/AGITG CO.17. Acta Oncol. 2014;53(7):877-884.

3. Vahabzadeh-Hagh AM, Rwigema JM, Nabili V, Wang MB, Lorentz WC. Predictors of prolongation in radiation treatment time in a veteran population treated with chemoradiation for oropharyngeal cancer. Acta Otolaryngol. 2018;138(1):80-84.

4. Waissbluth S, Peleva E, Daniel SJ. Platinum-induced ototoxicity: a prevailing ototoxicity criteria. Eur Arch Otorhinlaryngol. 2017;274(3):1187-1196.

5. Huang J, Zhang J, Shi C, Liu L, Wei Y. Survival, recurrence and toxicity of HNSCC in comparison of a radiotherapy combination with cisplatin versus cetuximab: a meta-analysis. BMC cancer. 2016;16(1):689-713.

6. Mittman N, Seung SJ. Rash rates with EGFR inhibitors: meta-analysis. Curr Oncol. 2011;18(2):e54-e63.

7. Boone SL, Rademaker A, Liu D, Pfeiffer C, Mauro DJ, Lacouture ME. Impact and management of skin toxicity associated with antiepidermal growth factor receptor therapy: survey results. Oncology. 2007;72(3-4):152-159.

8. Erbitux (cetuximab). Ask Lilly website. www.erbitux.com/hcp/index. html. Updated July 3, 2018. Accessed November 27.

9. Busam KJ, Capodieci P, Motzer R, Kiehn T, Phelan D, Halpern AC. Cutaneous side-effects in cancer patients treated with antiepidermal growth factor receptor antibody C225. Br J Dermatol. 2001;144(6):1169-1176.

10. Agero AL, Dusza SW, Benvenuto-Andrade C, Busam KJ, Myskowski P, Halpern AC. Dermatologic side effects associated with the epidermal growth factor receptor inhibitors. J Am Acad Dermatol. 2006;55:657-670, 2006.

11. Bernier J, Bonner J, Vermorken JB, et al. Consensus guidelines for the management of radiation dermatitis and coexisting acne-like rash in patients receiving radiotherapy plus EGFR inhibitors for the treatment of squamous cell carcinoma of the head and neck. Ann Oncol. 2008;19(1):142-149.

12. Burtness B, Anadkat M, Basti S, et al. NCCN task force report: management of dermatologic and other toxicities associated with EGFR inhibition in patients with cancer. J Natl Compr Canc Netw. 2009; 7(suppl 1):S5-S21.

13. Cabezón-Gutierrez L, Khosravi-Shahi P, Escobar-Álvarez Y. Management of dermatitis in patients with locally advanced squamous cell carcinoma of the head and neck receiving cetuximab and radiotherapy. Oral Oncol. 2012;48(4):293-297.

14. Li T, Perez-Soler R. Skin toxicity associated with epidermal growth factor receptor inhibitors. Target Oncol. 2009;4(2):107-119.

15. Bernier J, Russi EG, Homey B, et al. Management of radiation dermatitis in patients receiving cetuximab and radiotherapy for locally advanced squamous cell carcinoma of the head and neck: proposals for a revised grading system and consensus management guidelines. Ann Oncol. 2011;22(10):2191-2200.

16. Zhu G, Lin JC, Kim SB, Bernier J, et al. Asian expert recommendation on management of skin and mucosal effects of radiation, with or without the addition of cetuximab or chemotherapy, in treatment of head and neck squamous cell carcinoma. BMC Cancer. 2016;16:42-62.

17. Pinto C, Barone CA, Girolomoni G, et al. Management of skin reactions during cetuximab treatment in association with chemotherapy or radiotherapy: update of the Italian expert recommendations. Am J Clin Oncol. 2016;39(4):407-415.

18. Eriksen JG, Kaalund I, Clemmensen O, Overgaard J, Pfeiffer P. Placebo-controlled phase II study of vitamin K3 cream for the treatment of cetuximab-induced rash. Support Care Cancer. 2017;25(7):2179-2185.

19. Watanabe S, Nakamura M, Takahashi H, et al. Dermopathy associated with cetuximab and panitumumab: investigation of the usefulness of moisturizers in its management. Clin Cosmet Investig Dermatol. 2017;10:353-361. 